\title{
PATRIMÔNIO PÉTREO PAULISTANO: ANÁLISE SOB A ÓTICA DE PROPAGAÇÃO DE ONDAS ULTRASSÔNICAS
}

STONE HERITAGE OF THE CITY OF SÃO PAULO: ANALYSIS FROM THE POINT OF VIEW OF ULTRASOUND PULSE VELOCITY

Eliane Aparecida Del Lama Alexander Martin Silveira Gimenez Danielle Grossi Diego Ferreira Ramos Machado Luciane Kuzmickas Natália Mendonça Rodrigues

Como citar este artigo:

DEL LAMA, Eliane Aparecida; GIMENEZ, Alexander Martin Silveira; GROSSI, Danielle; MACHADO, Diego Ferreira Ramos; KUZMICKAS, Luciane; RODRIGUES, Natália Mendonça. Patrimônio pétreo paulistano: análise sob a ótica de propagação de ondas ultrassônicas. In: Cadernos do Lepaarq, v. XV, n.30, p. 229-236, Jul-Dez, 2018. 


\title{
Patrimônio pétreo paulistano: análise sob a ótica de propagação de ondas ultrassônicas
}

\author{
Eliane Aparecida Del Lama* \\ Alexander Martin Silveira Gimenez* \\ Danielle Grossi* \\ Diego Ferreira Ramos Machado* \\ Luciane Kuzmickas* \\ Natália Mendonça Rodrigues*
}

Resumo: A medição da velocidade de propagação de ondas ultrassônicas, método de análise não destrutivo, é uma técnica eficiente para avaliar o estado de conservação da rocha e a profundidade de penetração de consolidantes em monumentos. Este trabalho objetiva analisar monumentos pétreos da cidade de São Paulo, com base nesta técnica. A superfície de acabamento é um ponto chave na obtenção dos dados, uma vez que superfícies polidas permitem o perfeito acoplamento dos transdutores, favorecendo a execução do ensaio de forma adequada. Os dados apontam rochas não alteradas ou ausência de fraturamento nos monumentos analisados.

\section{Palavras Chave:}

Ultrassom; Monumentos; Métodos não Destrutivos;

Patrimônio Pétreo.

\begin{abstract}
Ultrasound pulse velocity, a non-destructive analytical method, is an efficient technique to evaluate the state of conservation of the rock and the depth of penetration of consolidants in monuments. This work aims to analyze stone monuments of the city of São Paulo based on this method. The finishing surface is a key point in obtaining the data, since polished surfaces allow the perfect coupling of the transducers, favoring the performance of the test in an appropriate manner. The data indicate unweathered rocks or absence of fractures in the analyzed monuments.
\end{abstract}

\section{Keywords:}

Ultrasound; Monuments; Non-destructive Methods; Stone Heritage.

\footnotetext{
* Instituto de Geociências da Universidade de São Paulo (USP), Brasil, Programa de Pós-Graduação Geociências (Mineralogia e Petrologia), Apoio da Fundação de Amparo à Pesquisa do Estado de S. Paulo (Fapesp), Brasil; (proc. n.
} 2015/10.858-8). E-mail: edellama@usp.br 


\section{INTRODUÇÃO}

Métodos não destrutivos são muito adequados para se trabalhar com o patrimônio histórico, uma vez que as análises podem ser realizadas in situ e sem sua fragmentação para coleta de amostras.

Vários são os métodos usados na inspeção de bens do patrimônio pétreo, tais como: determinação da velocidade de propagação de ondas ultrassônicas para avaliar a porção interna da rocha, determinação de cor por espectrofotometria, correlação da resistência por esclerometria, absorção de água com tubo de Karsten, digitalização térmica para detectar cavidades, tomografia computadorizada para inspeção de defeitos internos, espectroscopia Raman para a identificação de compostos químicos, obtenção de imagem por radiação ultravioleta para identificação de anomalias, fluorescência de raios X portátil para detecção de elementos químicos, laser scanner 3D, entre outros.

Estudos de caracterização da rocha por métodos não destrutivos já foram realizados em alguns monumentos da cidade de São Paulo (AUGUSTO, 2009; RODRIGUES, 2012; KUZMICKAS; DEL LAMA, 2014; GIMENEZ; DEL LAMA, 2014; GROSSI; DEL LAMA, 2015; GROSSI et al., 2015; MACHADO, 2015; DEL LAMA et al., 2016).

Este trabalho enfoca os resultados dos ensaios realizados, referentes às velocidades de propagação de ondas ultrassônicas, nas rochas utilizadas no patrimônio paulistano.

\section{MÉTODO DO ULTRASSOM}

A determinação da velocidade das ondas ultrassônicas permite a avaliação da sanidade da rocha e a identificação de fraturas profundas e superficiais, quando existentes.

Por ser de fácil operação e portabilidade, o método é adequado para análises diretas em monumentos ou, até mesmo, em amostras no laboratório.

O método foi desenvolvido originalmente para análise de concreto, sendo usado desde 1920.

As normas para os ensaios em concreto são: ABNT NBR 8802/94 (ABNT, 1994), BS EN 12504-4 (BS, 2004) e ASTM C-597/2016 (ASTM, 2016). A determinação da velocidade ultrassônica em rochas baseia-se na norma técnica ASTM D 2845/08 (ASTM, 2008).

A velocidade ultrassônica depende da densidade e das propriedades elásticas do material em questão.

O equipamento gera pulsos ultrassônicos de baixa frequência, medindo o tempo que estes levam para atravessar o material de um transdutor emissor para o transdutor receptor.

No ensaio, a distância percorrida pelas ondas ultrassônicas corresponde ao espaçamento entre os transdutores e o tempo de percurso é obtido no equipamento, e, desta forma, obtém-se a velocidade do ultrassom atravessando o material ensaiado, pela fórmula: velocidade $=$ distância/tempo.

Velocidades altas indicam que a rocha não apresenta alteração, sendo que velocidades baixas correlacionam-se com rocha intemperizada ou rocha fraturada.

Há 3 formas para se realizar o ensaio: transmissão direta (os transdutores são colocados em faces opostas do material), transmissão semidireta (os transdutores são colocados em faces adjacentes) e transmissão indireta ou superficial (os transdutores são colocados na mesma face).

Especificamente para determinação de medição de velocidades ultrassônicas em monumentos, Gimenez e Del Lama (2014) apresentam uma proposta. 
Por ser não invasivo e sua simplicidade de utilização, o método do ultrassom é muito usado na caracterização da rocha em monumentos no mundo todo (ALMESBERGER et al., 2000; FITZNER, 2004; SILVA, 2005).

O método do ultrassom também é usado para indicar a profundidade de penetração de produtos utilizados no tratamento de rocha (DELGADO RODRIGUES et al., 1996; ÁLVAREZ et al., 2008; PAMPLONA et al., 2008; SCHERER; JIMÉNEZ-GONZÁLEZ, 2008; FERREIRA PINTO; DELGADO RODRIGUES, 2012). Costa e Delgado Rodrigues (2008) indicam o ultrassom como o melhor instrumento para medir a eficácia dos consolidantes em rochas graníticas.

\section{MATERIAIS E MÉTODOS}

Para a execução de medidas ultrassônicas em monumentos da cidade de São Paulo, foi utilizado o aparelho V-Meter III da James Instruments Inc. e transdutores planos de $54 \mathrm{kHz}$ e $150 \mathrm{kHz}$.

As medidas foram realizadas em laboratório, em amostras coletadas em pedreiras e posteriormente serradas e, também, in situ, em monumentos e edifícios, onde foram selecionadas as porções mais planas e menos rugosas para facilitar o acoplamento dos transdutores.

Além dos monumentos do centro velho (Sé, Camões, Mário de Andrade, Biblioteca Mário de Andrade, José Bonifácio), foram analisados monumentos no Cemitério da Consolação (O Sepultamento), no bairro do Jardim Paulistano (Monumento em Homenagem ao Club Atlético Paulistano), defronte ao Parque do Ibirapuera (Monumento às Bandeiras) (Figura 1), defronte ao Jóquei Club de São Paulo (Nostalgia) e na Cidade Universitária (Monumento a Ramos de Azevedo).

Nos ensaios, foram selecionados monumentos representativos do patrimônio paulistano, quais sejam: Catedral Metropolitana, ou simplesmente Sé (Granito Itaquera, Granito Cinza Mauá, Granito Preto Piracaia, Giallo di Siena, Carrara, Verde Saint-Denis e Vermelho Portasanta); Nostalgia, Monumento em Homenagem ao Club Atlético Paulistano, O Sepultamento e Monumento a Ramos de Azevedo (Granito Itaquera); Camões, Biblioteca Mário de Andrade e Monumento às Bandeiras (Granito Cinza Mauá); e José Bonifácio (Granito Verde Ubatuba).

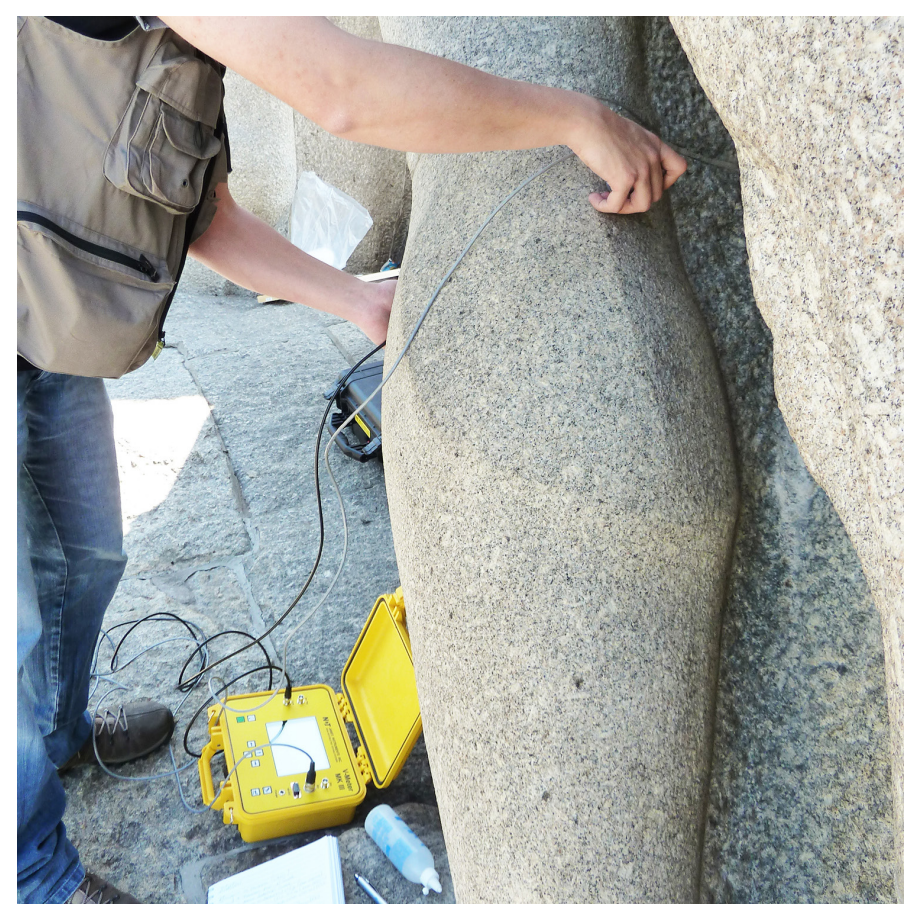

Figura 1. Medição da velocidade de propagação de ondas ultrassônicas pelo método direto no Monumento às Bandeiras. 


\section{RESULTADOS}

Os dados de medição das velocidades de propagação de ondas ultrassônicas, obtidos nas rochas utilizadas no patrimônio paulistano, estão apresentados na Tabela 1, tanto in situ quanto em laboratório.

É importante ressaltar que os valores obtidos dependem muito da acoplagem dos transdutores nas superfícies analisadas. A acoplagem em superfícies polidas geralmente é perfeita, sendo os valores de velocidade superiores àqueles obtidos em superfície serrada e/ou outros tipos de superfícies mais irregulares.

O Granito Itaquera apresenta valores de 4,1 a 5,0 km/s para a rocha fresca. Dos monumentos analisados, apenas o Monumento a Ramos de Azevedo e a base da esfera armilar do Monumento em Homenagem ao Club Atlético Paulistano apresentam valores compatíveis com a rocha sã. Neste último monumento, também foram encontrados os menores valores nas pilastras do banco, local onde há constantemente fogueira acendida por moradores de rua, o que deve ter diminuído a resistência da rocha. Na obra $O$ Sepultamento, os valores inferiores foram obtidos em suas bordas e locais afetados por alteração cromática e colonização biológica.

O Granito Cinza Mauá apresenta valores de 4,3 a 5,6 km/s para a rocha sã. Com exceção da estátua de Camões, os monumentos analisados estão dentro da faixa da rocha fresca. O valor acima desta faixa encontrada na Biblioteca Mário de Andrade foi obtido em superfície polida.

O Granito Verde Ubatuba apresenta valores de 4,1 a $4,5 \mathrm{~km} / \mathrm{s}$ e a base do monumento de José Bonifácio apresenta valores de 6,0 a $6,6 \mathrm{~km} / \mathrm{s}$, obtidos em superfícies polidas.

O Granito Preto Piracaia foi medido apenas na Sé, com valor de 4,1 km/s. Para o Serpentinito Verde Saint-Denis, também foi feita a medição em apenas um ponto, com valor de $4,3 \mathrm{~km} / \mathrm{s}$. Considerando-se que ambas as superfícies analisadas são polidas, os valores estão um pouco baixos, implicando em sanidade inferior para estes materiais.

Os mármores analisados apresentam valores acima de $5,1 \mathrm{~km} / \mathrm{s}$. Mármores sem alteração normalmente apresentam esta velocidade, e aqui se adiciona ainda que as superfícies medidas são polidas e localizam-se em ambientes internos, o que favorece a preservação de sua integridade.

\section{CONSIDERAÇÕES FINAIS}

No geral, os monumentos analisados apresentam-se internamente sem alteração, com base nos valores das velocidades de propagação de ondas ultrassônicas obtidos.

Os dados aqui obtidos permitem inferir que velocidades acima de 4,0 km/s em superfícies serradas ou suavizadas indicam rocha sã.

O acabamento da superfície analisada é essencial na obtenção destes dados, assim, para utilizar-se o método ultrassônico com eficiência, é necessário comparar dados obtidos nas mesmas condições, e não considerar apenas valores absolutos. 
Tabela 1. Valores de velocidade de propagação de ondas ultrassônicas de rochas utilizadas no patrimônio paulistano.

\begin{tabular}{|c|c|c|c|}
\hline Litotipo & Local de ensaio & $\begin{array}{l}\text { Superfície } \\
\text { analisada }\end{array}$ & $\begin{array}{c}\text { Velocidade } \\
(\mathrm{km} / \mathbf{s})\end{array}$ \\
\hline \multirow{9}{*}{ Granito Itaquera } & Laboratório (1) & Serrada & $4,1-5,0$ \\
\hline & Nostalgia (1) & Serrada & $3,4-4,1$ \\
\hline & $\begin{array}{l}\text { Mon. em Hom. ao Club Atlético Paulistano (base da } \\
\text { esfera armilar) (1) }\end{array}$ & Serrada & $4,2-4,7$ \\
\hline & $\begin{array}{l}\text { Mon. em Hom. ao Club Atlético Paulistano (pilastras do } \\
\text { banco) (1) }\end{array}$ & Serrada & $2,5-3,7$ \\
\hline & Laboratório (2) & Serrada & $4,1-4,5$ \\
\hline & O Sepultamento (3) & Irregular & $3,1-4,4$ \\
\hline & Monumento a Ramos de Azevedo (4) & Apicoada & $4,2-5,0$ \\
\hline & Laboratório (4) & Serrada & $4,2-4,8$ \\
\hline & Sé (5) & Serrada & 3,8 \\
\hline \multirow{7}{*}{ Granito Cinza Mauá } & Laboratório (1) & Serrada & $4,3-5,6$ \\
\hline & Camões (1) & Serrada & $3,3-5,1$ \\
\hline & Biblioteca Mário de Andrade (1) & Polida & $4,7-5,7$ \\
\hline & Mário de Andrade (1) & Serrada & $4,5-4,7$ \\
\hline & Monumento às Bandeiras (6) & Irregular & $4,6-5,3$ \\
\hline & Laboratório (6) & Serrada & $5,2-5,6$ \\
\hline & Sé (5) & Serrada & $4,4-5,5$ \\
\hline \multirow{2}{*}{ Granito Verde Ubatuba } & Laboratório (1) & Serrada & $4,1-4,5$ \\
\hline & José Bonifácio (1) & Polida & $6,0-6,7$ \\
\hline Granito Preto Piracaia & Sé (5) & Polida & 4,1 \\
\hline Giallo di Siena & Sé (5) & Polida & $5,7-5,9$ \\
\hline Carrara & Sé (5) & Polida & 6,2 \\
\hline Verde Saint-Denis & Sé (5) & Polida & 4,3 \\
\hline Vermelho Portasanta & Sé (5) & Polida & 5,1 \\
\hline
\end{tabular}

Fonte: (1) Rodrigues (2012), (2) Kanke (2013), (3) Kuzmickas e Del Lama (2014), (4) Grossi e Del Lama (2015), (5) Machado (2015), (6) Gimenez e Del Lama (2014).

A obtenção de dados ultrassônicos, por se tratar de uma técnica de análise do tipo não destrutivo, é uma ferramenta útil e adequada para a avaliação do estado de conservação de monumentos, podendo ser aplicada no patrimônio pétreo sem reservas. A partir deste diagnóstico, há a possibilidade de ser indicadas e implementadas medidas e formas para recuperação e mitigação de danos nos monumentos alterados, assim como para a própria verificação da eficácia de técnicas de tratamento, como a aplicação de consolidantes. Os trabalhos preventivos com essa ótica podem ser executados em prazos curtos, a um custo mínimo.

\section{Agradecimentos}

À Fapesp (Fundação de Amparo à Pesquisa no Estado de São Paulo) pela concessão do auxílio à pesquisa (proc. 2015/10858-8). 


\section{REFERÊNCIAS}

ABNT - ASSOCIAÇÃO BRASILEIRA DE NORMAS TÉCNICAS. NBR 8802: Concreto endurecido - Determinação da velocidade de onda ultra-sônica. Rio de Janeiro, 1994.

ALMESBERGER, Dario; GEOMETRANTE, Raffaella; RIZZO, Antonio; SURAN, P. Ultrasonic testing method for the characterization of Pietra D'Istria structural elements. In: INTERNATIONAL CONGRESS ON DETERIORATION AND CONSERVATION OF STONE, 9., 2000, Venice. Proceedings... Venice, 2000. v. 2, p. 317-325.

ÁLVAREZ, Araceli R.; REDONDO, Félix M.; MENÉNDEZ, Luis V. 2008. Consolidation of granite building stones used in continental climates: San Juan de los Reyes Church in Toledo, Spain. In: INTERNATIONAL SYMPOSIUM STONE CONSOLIDATION IN CULTURAL HERITAGE - RESEARCH AND PRACTICE, 2008, Lisbon. Proceedings... Lisbon: LNEC, 2008. p. 213-222.

ASTM - AMERICAN SOCIETY FOR TESTING AND MATERIALS. D-2845: Standard method for laboratory determination of pulse velocities and ultrasonic elastic constants of rock. Philadelphia, 2008.

ASTM - AMERICAN SOCIETY FOR TESTING AND MATERIALS. C-597: Standard test method for pulse velocity through concrete. West Conshohocken (PA, EUA), 2016.

AUGUSTO, Wilian C.B. Caracterização geológica dos monumentos da cidade de São Paulo. Monografia de Trabalho de Formatura, Instituto de Geociências, Universidade de São Paulo (IGc-USP), 2009.

BS - BRITISH STANDARDS. BS EN 12504: Testing concrete. Determination of ultrasonic pulse velocity. 2004.

COSTA, Dória; DELGADO RODRIGUES, José. Evaluation of consolidation treatments applied to granitic materials. Experience and critical overview of laboratory testing. In: INTERNATIONAL SYMPOSIUM STONE CONSOLIDATION IN CULTURAL HERITAGE - RESEARCH AND PRACTICE, 2008, Lisbon. Proceedings... Lisbon: LNEC, 2008. p. $389-398$.

DEL LAMA, Eliane A.; DEHIRA, Lauro K.; GROSSI, Danielle; KUZMICKAS, Luciane. The colour of the granite that built the city of São Paulo, Brazil. Color Research and Application, 41(3), p. 241-245, 2016.

DELGADO RODRIGUES, José; COSTA, Dória; SCHIAVON, Nick. Spatial distribution in granite stones. In: DELGADO RODRIGUES, José; COSTA, Dória. (Ed.). Conservation of granitic rocks. 1st ed. Lisbon: LNEC, 1996. p. 55-61.

FERREIRA PINTO, Ana P.; DELGADO RODRIGUES, José. Consolidation of carbonate stones: Influence of treatment procedures on the strengthening action of consolidants. Journal of Cultural Heritage, 13, p. 154-166, 2012.

FITZNER, Bernd J. Documentation and evaluation of stone damage on monuments. In: INTERNATIONAL CONGRESS ON DETERIORATION AND CONSERVATION OF STONE, 10., 2004, Stockholm. Proceedings... Stockholm, 2004. p. 667-690.

GIMENEZ, Alexander M.S.; DEL LAMA, Eliane A. Comportamento de ondas ultrassônicas no Granito Mauá para a conservação do Monumento às Bandeiras. Geologia USP - Série Científica, Revista do Instituto de Geociências-USP, 14(3), p. 47-60, 2014. Disponível em: http://ppegeo.igc.usp.br/pdf/guspsc/v14n3/04.pdf.

GROSSI, Danielle; DEL LAMA Eliane A. Ultrasound technique to assess the physical conditions of the Monument to Ramos de Azevedo. Revista Escola de Minas (REM), 68(2), p. 171-176, 2015. Disponível no endereço: http://www.scielo. br/pdf/rem/v68n2/0370-4467-rem-68-02-0171.pdf. 
GROSSI, Danielle; DEL LAMA, Eliane A.; GARCIA-TALEGON, Jacinta; IÑIGO, Adolfo C.; VICENTE-TAVERA, Santiago. Evaluation of colorimetric changes in the Itaquera Granite of the Ramos de Azevedo Monument, São Paulo, Brazil. International Journal of Conservation Science, 6, p. 313-322, 2015. Disponível em: http://ijcs.uaic.ro/public/ IJCS-15-29_Grossi.pdf.

KANKE, Rafael A. Utilização do Granito Itaquera em obras históricas do centro da cidade de São Paulo. Monografia de Trabalho de Formatura, Instituto de Geociências, Universidade de São Paulo (IGc-USP), 2013.

KUZMICKAS, Luciane; DEL LAMA, Eliane A. Utilização de métodos não destrutivos no patrimônio histórico: estudo de caso da escultura O Sepultamento de Victor Brecheret. Revista Brasileira de Geologia de Engenharia e Ambiental (RBGEA), 4(1), p. 9-22, 2014. Disponível em: http://www.abge.org.br/uploads/arquivos/artigo1-dellama2015100813351380545.pdf.

MACHADO, Diego F.R. Catedral da Sé de São Paulo: análise do estado de conservação das rochas do templo por métodos não destrutivos. Dissertação de Mestrado, Instituto de Geociências, Universidade de São Paulo (IGc-USP), 2015. Disponível em: http://www.teses.usp.br/teses/disponiveis/44/44144/tde-27052015-090139/.

PAMPLONA, Marisa; KOCHER, Mathias; SNETHLAGE, Rolf; WENDLER, Eberhard. Consolidation effectiveness of TEOS on Ançã limestone from Portugal - A laboratory study. In: INTERNATIONAL SYMPOSIUM STONE CONSOLIDATION IN CULTURAL HERITAGE - RESEARCH AND PRACTICE, 2008, Lisbon. Proceedings... Lisbon: LNEC, 2008. p. 183-192.

RODRIGUES, Natália M. Ensaios não destrutivos em monumentos pétreos paulistanos. Monografia de Trabalho de Formatura, Instituto de Geociências, Universidade de São Paulo (IGc-USP), 2012.

SCHERER, George W.; JIMÉNEZ-GONZÁLEZ, Inmaculada. 2008. Swelling clays and salt crystallization: damage mechanisms and the role of consolidants. In: INTERNATIONAL SYMPOSIUM STONE CONSOLIDATION IN CULTURAL HERITAGE - RESEARCH AND PRACTICE, 2008, Lisbon. Proceedings... Lisbon: LNEC, 2008. p. 29-39.

SILVA, Luiz C.T.da. Avaliação da degradação de rochas em fachadas de prédios históricos: Os exemplos do Teatro Municipal e do Paço Imperial, Cidade do Rio de Janeiro. Dissertação (Mestrado), Instituto de Geociências, Universidade Federal do Rio de Janeiro, 2005.

Recebido em: 30/01/2018

Aprovado em: 30/05/2018

Publicado em: 30/11/2018 